\title{
Entrelacs
}

Cinéma et audiovisuel

$6 \mid 2007$

L'Arbre

\section{Les arbres : des hommes comme les autres}

Figures d'arbres : à propos de Dersou Ousala (Akira Kurosawa, 1975)

Valérie Zanello

\section{(2) OpenEdition}

Journals

Édition électronique

URL : http://journals.openedition.org/entrelacs/321

DOI : $10.4000 /$ entrelacs.321

ISSN : 2261-5482

Éditeur

Éditions Téraèdre

Édition imprimée

Date de publication : 1 mars 2007

Pagination : 35-44

ISBN : 978-2-912868-70-1

ISSN : 1266-7188

Référence électronique

Valérie Zanello, "Les arbres: des hommes comme les autres », Entrelacs [En ligne], 6 | 2007, mis en ligne le 01 août 2012, consulté le 22 avril 2019. URL : http://journals.openedition.org/entrelacs/321 . DOI : 10.4000/entrelacs.321

Ce document a été généré automatiquement le 22 avril 2019.

Tous droits réservés 


\section{Les arbres : des hommes comme les autres}

Figures d'arbres : à propos de Dersou Ousala (Akira Kurosawa, 1975)

Valérie Zanello

1 Après l'échec public et critique de Dodes'kaden (Dodesukaden, 1970), des ennuis de santé et une tentative de suicide, Akira Kurosawa, cinéaste au perfectionnisme légendaire confinant parfois au pathologique, réalise en 1975 Dersou Ouzala (Derusu Uzara). Ce film ne marque pas seulement le retour du cinéaste derrière la caméra après cinq longues années de silence, il affirme aussi un changement radical en regard de l'univers du film précédent : la Russie plutôt que le Japon, la taïga plutôt que les bidonvilles, l'ouverture plutôt que l'enfermement ... et les arbres vivants plutôt que l'unique arbre mort de Dodes'kaden. Expression d'un élan vital, Dersou Ouzala renoue ainsi avec le monde foisonnant et labyrinthique des forêts du Château de l'Araignée et de Rashômon.

Dersou Ouzala est l'adaptation d'un récit, très populaire en Russie, de Vladimir Arseniev ${ }^{1}$, un officier du tsar chargé d'explorer, entre 1902 et 1910, la région de l'Oussouri, située aux confins de la Sibérie et de la Chine, et limitée à l'est par la mer du Japon. Dans cette partie extrême du monde où le cinéaste a tourné l'essentiel de son film, on rencontre la faune et la flore propre à la taïga sub-arctique. Cette végétation s'étend le long de vastes vallées fluviales et de deltas qui le disputent à un paysage constitué de gigantesques forêts feuillues et mixtes encombrées de lianes.

3 La nature dans Dersou Ouzala revêt une fonction et une valeur tout à fait particulières. Loin de servir de simple décor au film, elle constitue en effet un sujet à part entière. Très tôt, Dersou, le chasseur golde dont Vladimir Arseniev a fait le héros de ses récits, nous apprend que la taïga est peuplée d'êtres très différents : pour lui, les animaux sont des " gens » ou des " personnes ", des « hommes " ${ }^{2}$, au même titre que les autres végétaux présents dans ce milieu naturel. Ainsi parle-t-il au feu, qui lui répond, à Amba le tigre ou au bois qui brûle. Même le bâton bifide sur lequel il s'appuie représente à ses yeux une personne. Quant aux arbres, ils sont à l'image même de cette communion de Dersou avec la nature, ainsi que le suggère la force stylistique de la caméra de Kurosawa qui les traite 
comme de véritables objets cinématographiques. Ainsi les arbres définissent-ils la place de l'homme, à la fois au sein de la nature, et dans l'espace délimité par le cadre de la caméra.

\section{Voiler et dévoiler : l'arbre-rideau, l'arbre-paravent}

Dans ce film, nombreux sont les plans où le feuillage des arbres apparaît au premier plan comme un rideau troué à travers lequel la scène est observée. En fait, les personnages (Dersou, Arseniev, les soldats) sont bien plus souvent vus qu'ils ne regardent : rares, en effet, sont les raccords sur leur regard, et nous sommes davantage en situation de les observer à travers les branches, que de regarder ce qu'ils voient. Les branches constituent tout un entrelacs de rameaux et de feuillages : les premiers dessinent ainsi des stries qui lacèrent le cadre et les personnages, tandis que les feuilles sont autant de taches qui brouillent l'image ocellée comme un pelage de fauve. La série de plans sur Amba, le tigre qui poursuit Dersou, synthétise ces deux procédés : stries et taches se fondent dans la robe du fauve, la confusion étant soulignée par un voile rouge lui-même lacéré qui recouvre l'image. Certes, ces arbres-rideaux ne sont pas nouveaux chez le cinéaste ; ils constituent même sa signature visuelle, surtout lorsque cette figure est associée au travelling latéral comme on peut le voir dans la forêt labyrinthique du Château de l'Araignée. Le rideau devient alors un long paravent plus ou moins opaque, à travers lequel nous observons, cachés, la course effrénée des personnages. Pour autant qu'ils masquent, les arbres-rideaux de Dersou Ouzala revêtent néanmoins une autre fonction. Ils intègrent en effet les êtres humains dans une nature enchevêtrée, désordonnée, chaotique, et échappant à toute forme de hiérarchisation. Proprement (re)mis à sa place, l'homme semble ainsi considéré au même titre que tous les êtres qui peuplent la taïga. Le procédé de l'arbre-rideau constitue, par ailleurs, une manière de pénétrer dans l'épaisseur de la taïga, qui est alors traitée comme une matière. À ce titre, l'entrée dans le film, après le générique, se fait derrière un rideau de feuilles qui dévoile l'avancée des soldats face au spectateur placé, de ce fait, derrière le rideau et dans l'épaisseur de la taïga.

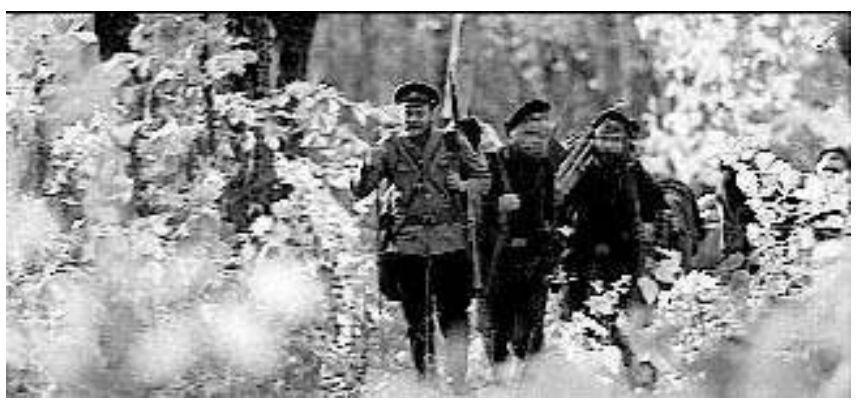

Si l'entrée dans le film est logiquement marquée par l'ouverture du rideau de feuilles, plus loin, c'est sa fermeture qui souligne l'engagement des personnages dans l'épaisseur de la forêt et dans le cœur de l'intrigue. En effet, lorsque Dersou, après avoir accepté de servir de guide à Arseniev, remet l'équipage dans le droit chemin, il s'engage sur une sente en sortant du cadre par le bord supérieur gauche. La caméra s'éloigne en un discret travelling arrière pour laisser passer les soldats et les chevaux ; l'image est alors voilée par le feuillage des arbustes qui vient occuper le premier plan. Comme un paravent troué, la taïga se referme sur les hommes dont Dersou est le guide, les englobant désormais dans sa profondeur. L'usage des focales longues permet également au cinéaste de travailler l'espace de la taïga comme une matière dense et sans profondeur de champ : entrelacs de branchages au premier plan, les êtres humains et la forêt sont alors pris dans un espace 
dénué de profondeur et dans lequel la vue est bloquée par un mur d'arbres ou de taillis. L'homme n'y a que peu de marge de manœuvre ; toute sa personne est mise à mal par la lacération des branches (l'arbre-rideau) et les murs d'arbres qui se dressent autour de lui. Dans cette nature plus indifférente que réellement hostile, l'homme doit se frayer un passage, affirmer sa place. Il est loin d'en constituer le centre ou la valeur absolue. La taïga comme matière visuelle et objet cinématographique, est la représentation concrète des paroles de Dersou : l'homme n'est qu'un élément de la nature, il est pris dans un réseau de relations qui unissent les nombreux " habitants " de la forêt, les animaux certes, mais aussi les végétaux, les minéraux ou les éléments naturels tels que le feu, le vent, ou encore la pluie, qui s'y manifestent.

\section{Tracer des frontières : l'arbre-seuil}

6 Lieu de communication entre la terre et le ciel, entre le royaume des morts et le monde des vivants, "Celui de qui la tête au ciel était voisine / Et dont les pieds touchaient à l'empire des morts $»^{3}$, selon les mots de La Fontaine, représente une figure cinématographique d'une extraordinaire richesse. L'arbre symbolise ainsi le franchissement d'un monde à l'autre et devient le seuil au-delà duquel on accède à un nouvel univers4. Cette figure de l'arbre-seuil apparaît dans Dersou Ouzala à plusieurs reprises. Destiné à être franchi, l'arbre revêt la forme d'une fourche bifide qu'on enjambe, d'un tronc couché, voire incliné, que les personnages décident parfois de franchir. Ainsi dans la séquence évoquée plus haut au sujet de l'arbre-rideau, lorsque Dersou, passant la porte symbolique de la forêt et du film, entraîne la troupe d'Arseniev derrière lui. La figure géométrique de l'arbre bifide ${ }^{5}$, dont la fourche dessine un $\mathrm{V}$ dans l'image, marque également le seuil entre deux univers dans une des séquences relatives à la première rencontre avec Amba le tigre. Le fauve reste invisible, seules ses traces ont permis à Dersou d'en déduire la présence. Alors que Dersou cherche à situer l'animal, un plan nous montre le Golde, armé de son fusil, encadré par les deux branches d'un tronc bifide en $\mathrm{V}$ qui nous fait face. De l'autre côté de ce seuil symbolique, que Dersou ne franchira pas, se trouvent l'âme de la taïga et le destin encore mystérieux que le personnage ne fait que pressentir.

Marquant une séparation entre deux espaces, l'arbre-seuil apparait, en outre rappelionsnous, couché ou incliné. Ainsi, dans la deuxième partie, quand Arseniev et Dersou se retrouvent dans la taïga, la progression des deux hommes l'un vers l'autre est rendue difficile par l'entrelacs des taillis. Ils finissent par se rejoindre, gênés toutefois dans leurs effusions par un énorme tronc d'arbre couché qui dessine une séparation; Arseniev se tient dans la moitié gauche du champ, alors que Dersou lui fait face de façon symétrique. Même si la dimension comique n'est pas totalement absente de ces retrouvailles entravées par la masse imposante de l'arbre, on peut se demander pourquoi le cinéaste signale le moment inaugural de la deuxième partie du film, par cet obstacle franchi tant bien que mal. C'est que ce seuil que l'amitié entre les deux hommes arrive à surmonter, marque en fait la séparation entre deux mondes, clivage que la suite du film ne fera que confirmer. Après ces retrouvailles conquises sur l'espace naturel, un long plan-séquence par exemple nous montre Arseniev et Dersou dans le coin inférieur droit de l'image, isolés du reste de la troupe par la souche et le tronc d'un arbre incliné, dont la masse noire traverse l'image dans sa diagonale ; les soldats sont nettement regroupés de l'autre côté 
de cette ligne noire ${ }^{6}$. Le seuil est seulement franchi par les voix des soldats qui chantent une belle mais moqueuse romance destinée au « couple » reconstitué.

L'arbre-seuil dessine ainsi la frontière entre un monde où l'homme vit en accord avec la nature, et des groupes humains de plus en plus importants ${ }^{7}$, les « maitres et possesseurs de la nature ".

\section{Verticalité des lignes : l'arbre-pilier : l'arbre-barreau}

Les populations de l'Oussouri oriental pratiquent le chamanisme, tradition religieuse de Sibérie et d'Asie centrale où l'arbre occupe une place centrale, notamment dans certains rites d'initiation.

"Le rite principal comporte une ascension. On fixe dans la yourte un bouleau solide, les racines dans l'âtre et le sommet sortant par le trou de fumée. Le bouleau est nommé udeshi burkhan, "le gardien de la porte ", car il ouvre au chaman l'entrée du Ciel. Il restera définitivement dans la tente pour servir de marque distinctive à la demeure du chaman. [...] Le bouleau ou le poteau sont assimilés à l'Arbre ou au Pilier qui se dressent au centre du monde et qui relient les trois zones cosmiques : Terre, Ciel, Enfers. $»^{8}$ Dans deux séquences majeures, les adieux du vieux Chinois et la nuit du Nouvel An, le piquet central de la tente d'Arseniev est nettement visible : il s'agit d'un bouleau. Cela ne fait évidemment pas du Capitaine un chaman. Mais la valeur symbolique et magique de l'arbre-pilier se trouve ainsi visuellement rappelée, avec d'autant plus de force qu'elle est récurrente dans tout le film. Certes, il peut paraître vain de souligner l'importance des lignes verticales dans un film qui se déroule essentiellement dans la forêt ! Cependant, il existe bien des façons de filmer une forêt, et toutes les forêts ne se ressemblent pas. Ainsi, tous ces plans où de fins troncs d'arbres noirs se dessinent sur le fond blanc de la neige comme autant de barreaux entre lesquels les personnages se déplacent, motifs qui ne le doivent qu'à la volonté du cinéaste, à ses choix impérieux puisque les séquences relatives à ces plans descriptifs, en effet n'apparaissent pas dans le livre d'Arseniev. Ou plutôt, lorsqu'elles y figurent, elles sont placées dans un contexte différent de celui du livre : la rencontre avec le vieux Chinois, dans la neige, n'est pas évoquée telle quelle dans le récit d'Arseniev ; elle semble plutôt être le condensé de plusieurs épisodes du livre. Or, dans cette séquence, nous relevons, outre les arbres évoqués précédemment, les piliers soutenant l'auvent de la cabane du vieux chinois, le piquet central de la tente d'Arseniev, et le bâton que Dersou remet au vieillard qui s'engage sur le chemin du retour vers son village après quarante ans de vie solitaire. De même, dans la deuxième partie du film, le moment où Dersou prend conscience de sa perte d'acuité visuelle se déroule sur un parterre enneigé au milieu de minces troncs d'arbres, ce qui n'est pas le cas dans le récit générique.

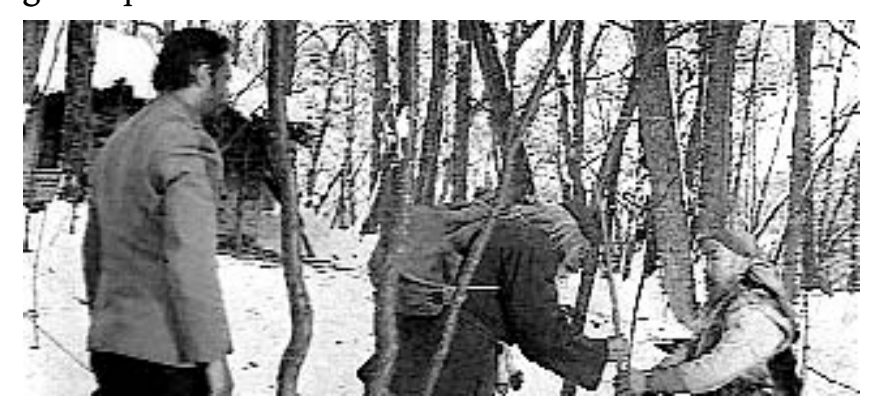


11 Les arbres-piliers servent de lien entre la terre et le ciel, mais filmés à hauteur d'homme sans que leur base et leur cime ne soient visibles ${ }^{9}$, ils deviennent autant de barreaux qui referment l'espace sur les personnages. Les deux séquences dont nous parlons se font écho esthétiquement (lignes noires verticales sur fond blanc), dramatiquement (abandon de la forêt par des hommes usés) et chronologiquement (au deux-tiers de chacune des deux parties).

12 Le pilier peut aussi se retrouver dans des objets fabriqués par l'homme, le piquet de tente, le fusil et le théodolite. Si le premier sert évidemment aux tentes qui protègent du vent et $\mathrm{du}$ froid, fusil et théodolite sont détournés de leur usage premier pour servir d'ossature à la hutte de roseaux improvisée par Dersou dans le terrible épisode du lac Khanka. Leur valeur symbolique et protectrice apparaît essentielle dans une nature qui n'est pas un Eden : Dersou doit chasser pour vivre et sait tirer quand il le faut, du moins jusqu'à sa faute fatale ; Arseniev sait mesurer et explorer le monde naturel pour mieux le comprendre, et pour, peut-être, mieux le dominer. Ces attributs propres à chacun des personnages trouvent leur place parmi les piliers que sont, par excellence, les arbres de la taïga. La valeur symbolique du théodolite est en outre confirmée par sa présence dans le très beau plan du coucher de soleil et du lever de lune ${ }^{10}$. Dans un des rares espaces ouverts du film, les deux silhouettes humaines sont encadrées par les deux astres fondateurs et garants de la survie du monde. À leur gauche se dresse le théodolite, emblème de la connaissance rationnelle et mathématique de ce même monde, attribut silencieux d'Arseniev qui écoute la parole inspirée de Dersou. De ce plan se dégage un grand sentiment d'harmonie : union des contraires, complémentarité de ce qui ne va pas l'un sans l'autre, nuit et jour, chaud et froid, science et animisme, chiffres et intuitions. En somme, Arseniev et Dersou.

13 Enfin, remarquons le pilier bifide qui parcourt le film : le bâton de Dersou, véritable attribut dont il ne sépare pas et que le Golde considère comme une personne à part entière. Son importance est soulignée dans le dernier plan du film, où la fourche du bâton de Dersou planté par Arseniev sur sa tombe, se dresse parmi les lignes verticales des troncs de la forêt, dont on sait qu'ils seront tous abattus, arbre parmi les arbres, lien entre la terre où repose le chasseur, et le ciel d'où tombent les flocons de neige. Pourquoi une si grande mélancolie se dégage-t-elle de ce plan si simple, si sobre ? D'abord parce que devinons-nous le destin du bâton de Dersou, ensuite parce que l'image de cette nature enneigée marque la fin d'un monde tout autant que la fin du film. Le bâton dressé devient une borne, la limite du film et de la taïga encore préservée. Dersou est enterré sur une frontière, entre l'espace sauvage de la forêt encore debout, et le talus de la voie ferrée. Cette borne sera abattue, comme les autres arbres.

\section{Ordre et désordre de la nature : l'arbre-chaos}

Abattis, rompis, chablis, loudéva , embûches ${ }^{11}$... Le récit de Vladimir Arseniev est émaillé de termes désignant du bois en désordre, des ensembles chaotiques de branches et de troncs, d'arbres effondrés, abattus ou déracinés. Dans chacune des deux parties du film, deux séquences majeures où les arbres ne sont plus piliers, où leur verticalité a été rompue, annoncent des moments de grand désordre. Ainsi, au début du film, alors que Dersou n'est pas encore arrivé, la troupe d'Arseniev s'arrête dans un chaos de pierres, de branchages et de troncs à terre ${ }^{12}$. Tous sont saisis par un étrange sentiment d'inquiétude irraisonnée. C'est pourtant là que le Capitaine décide de bivouaquer. Le désordre mental 
se manifestera par l'insomnie d'Arseniev et ses visions fantasmagoriques, interrompues par la venue de Dersou.

Toute aussi marquante est la séquence des loudeva. Ici, le chaos engendré par les hommes " mauvais ", comme le dit Dersou, est contre-nature : il bouleverse l'espace naturel parce que les pièges ne sont pas remblayés, il cause la mort inutile d'animaux dont la carcasse pourrira. Abattis d'arbres, abattoir d'animaux. C'est bien de la folie de l'homme dont il est question ici, de ses excès et de son irresponsabilité. Le cinéaste, dans son habituelle délicatesse, nous épargne la vision des charognes. Il préfère nous montrer le sauvetage aisé et rapide d'un jeune daim qu'Arseniev prend dans ses bras, en un geste très pastoral, et qu'il rend à sa liberté ${ }^{13}$. Plus loin, le plan-séquence qui nous donne une vue d'ensemble de l'abattis n'est pas interrompu par des inserts sur le carnage ; seules des voix hors-champ nous parviennent, décrivant ce qu'elles ont sous les yeux : « comme à l'abattoir ", "on se croirait chez un équarrisseur ", " rien que de la charogne ». La séquence se clôt sur Arseniev songeur, dans la même attitude qui fut la sienne à la fin du prologue. On devine l'interrogation d'un homme doutant désormais du bien-fondé de sa mission, habité par le pressentiment qu'une longue et inéluctable dégradation a commencé. Le chaos n'est donc pas tant l'expression d'une nature inquiétante et mystérieuse que la visualisation d'une nature en déséquilibre, nous voulons dire bouleversée par des hommes qui n'y sont pas à leur place. En fait, la taïga de Dersou n'offre d'espace à l'humain qu'à condition qu'il se fonde en elle, qu'il renonce à sa place de dominateur et qu'il accepte de n'en être qu'un élément parmi d'autres.

\section{Hallucinations : l'arbre magique}

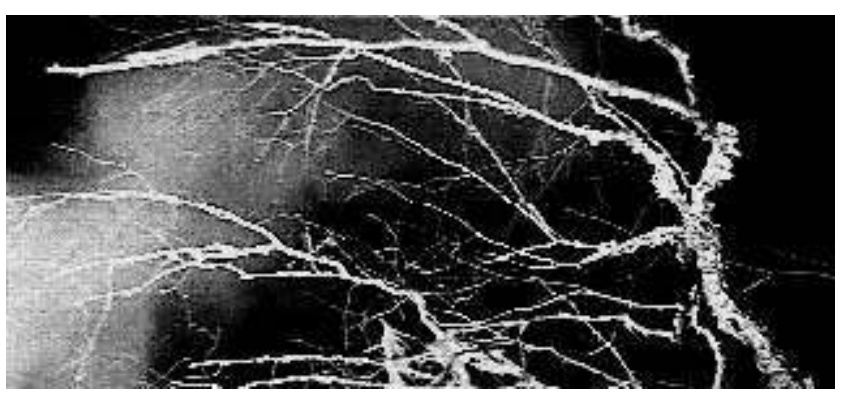

Présente dans le récit de Vladimir Arseniev, la taïga, étrange et parfois effrayante, donne lieu à une séquence au traitement cinématographique proprement stupéfiant. Il s'agit de l'insomnie d'Arseniev. Près du feu, le Capitaine rédige son carnet de bord en évoquant explicitement le climat magique qui habite le lieu et qui lui rappelle l'épisode de « La Nuit de Walpurgis $\aleph^{14}$. Tout un pan de la littérature populaire germanique fait dès lors irruption dans la taïga sibérienne, idée que l'on doit entièrement à l'imaginaire du cinéaste japonais. En effet, Vladimir Arseniev ne fait aucune allusion à la Nuit de Walpurgis dans ses écrits. S'il y a encore lieu de souligner l'immense culture cosmopolite de Kurosawa, saluons ici l'usage qu'il en fait dans des plans proprement fantastiques. Le bois qui brûle dans le feu du bivouac semble se tordre de douleur, les branches, surexposées par une étrange lumière, se dressent dans la nuit comme autant de bras tendus. Le bois, les arbres, le feu vivent. Toutefois, la présence de ces arbres magiques n'est pas fortuite, ni même uniquement esthétique. Les visions fantasmagoriques annoncent la rencontre avec Dersou qui parlera sans ambages aux flammes et aux bûches, les considérant comme des êtres à part entière, au même titre que le Capitaine et ses 
soldats. Ce que les légendes de l'Occident ont exprimé à travers l'art, Dersou le formule simplement, immédiatement, comme une évidence. Maintenant, la rencontre entre Arseniev et le Golde peut avoir lieu.

Si le chasseur de la taïga devient, logiquement ici, un double de Méphistophélès, ce n'est pas par sa dimension maléfique et diabolique, mais pour la fonction initiatique qu'il occupe. Dans le Faust de Goethe, Méphisto est aussi le guide du savant, du scientifique ${ }^{15}$ qui cherche à dominer le monde ; il est également celui qui permet à Faust d'accéder à son destin dans son désir de s'élever. "La Nuit de Walpurgis » constitue à ce titre, dans le deuxième Faust, un long rituel d'initiation occulte à la lumière des brasiers maléfiques. Or, il est vrai que le motif igné est récurrent dans le film, toujours associé à Dersou qui parle au feu et le personnifie, qui reste éveillé devant le foyer tout en communiquant avec sa famille défunte devant un brasier ; dans la maison d'Arseniev, il contemple le feu prisonnier du poêle, et c'est un feu de camp qui veille sur sa tombe. Mais DersouMéphisto n'est pas ici l'associé des flammes de l'Enfer ${ }^{16}$, il représente celui qui porte la flamme éclairante, la lumière qui réchauffe et protège.L'arbre magique ${ }^{17}$ tient naturellement sa place parmi les figures d'arbres convoquées par le film. Même le sapin de Noël, décoré de cuillères, de fourchettes métalliques et de glaçons tintinnabulant, devient inquiétant dans le blizzard de la nuit du Nouvel An. Il annonce aussi bien la venue extraordinaire d'Amba le tigre que le déclin de Dersou. Et pourtant, jamais le cinéaste n'insiste sur la dimension fantastique de la taïga. Dersou Ouzala n'est pas un film de genre. En outre, c'est toujours avec un très grand respect de la nature et des croyances des hommes que Kurosawa évite tout didactisme, tout moralisme, ou même toute récupération esthétique : aucun " message » écologiste militant, aucun discours orienté vers un animisme qui sauverait le monde, aucun engagement annonçant la mode « New Age ", aucun débordement vers une esthétique du fantastique. En effet, l'arbre magique, l'arbre-sorcier est toujours montré comme une projection de l'esprit des hommes ; la nature n'est ni bonne ni mauvaise, elle est ce que les hommes en font, objet d'art fantasmagorique pour Arseniev, objet d'une combinaison équilibrée pour Dersou, objet d'exploitation et de domination pour les autres.

\title{
l'arbre mort
}

\author{
«VLADIMIR : Il a dit devant l'arbre. (Ils regardent l'arbre.) Tu en vois d'autres ? \\ ESTRAGON : Qu'est-ce que c'est?

18 Samuel Beckett rappelle qu'en perdant ses feuilles, en perdant la vie (et ici, c'est à juste titre une vie de pleurs), l'arbre perd aussi son identité, son nom. Ce que nous dit l'épouse de Heï l'aveugle, dans Dosdeskaden, lorsqu'elle pose sa main sur le tronc de l'arbre mort (encore une figure bifide) qui se dresse, solitaire, dans le bidonville : «Cet arbre... Quel est son nom ? Quand un arbre est mort, il n'a plus de nom ». De même, dans le prologue de Dersou Ouzala qui montre comment le village des pionniers russes a fait disparaitre la tombe de Dersou et une partie de la forêt, Arseniev se souvient que se dressaient là un cèdre et un sapin. Le pionnier interrogé, quant à lui, a perdu les noms de ces autres " hommes " que sont les arbres. Les repères spatiaux et onomastiques ${ }^{19}$ ont totalement disparu. Demeure quand même, sur la tombe du Golde et dans notre mémoire, l'image 
ultime du bâton fourchu planté dans le sol, arbre parmi les arbres, au milieu d'une nature devenue sanctuaire. Dans le dernier plan, l'attribut de Dersou se dresse comme un arbre nu, alors que le nom du héros éponyme s'inscrit sur l'écran. Si la mort de Dersou marque la disparition de la nature, détruite par l'hybris des hommes, elle signe également la disparition du dernier chasseur golde de cette taïga sub-arctique. Au terme de ce magnifique voyage aux résonances cosmologiques évidentes, le cadavre, comme le bois débité des cèdres, des sapins ou du bâton fourchu, ont recouvré cette part humaine que Dersou reconnaissait à toutes les choses de la nature. Si l'on veut bien admettre, évidemment, l'origine étymologique d'humanus qui est humus, autrement dit, la terre.

\section{NOTES}

1. Vladimir Arseniev, Dersou Ouzala, traduction de Pierre P. Wolkonsky, Paris ?, Pygmalion, 2003.

2. Dans les sous-titres français de l'édition DVD (version originale russe, traduction d'Alexandre Karvovski, MK2 Éditions, 2005), Dersou utilise le mot "gens », alors que la version audio doublée parle plutôt de « personnes ». La traduction de Pierre P. Wolkonsky fait état du mot « hommes ». 3. Jean de La Fontaine, "Le Chêne et le Roseau», Fables, I, 22, vers 31-32, Paris, Classiques Garnier, 1962, p. 55.

4. L'arbre comme seuil n'est pas sans évoquer le torii, portique qui permet d'accéder à un temple bouddhiste. Dans les films de Miyazaki comme Mon voisin Totoro, Le Voyage de Chihiro, ou encore Princesse Mononoke, l'entrée dans l'autre monde se fait en passant par la forêt.

5. André Leroi-Gourhan donne une photographie d'un arbre sacré de ce type, dans Pages oubliées sur le Japon, recueil posthume établi par J.-F. Lesbre, Grenoble, Editions ? Jérôme Million, 2004, ill. 20 , page $\mathrm{V}$.

6. Cette analyse est développée par Stephen Rpice ??, The Warrior's camera, Princeton University Press, édition revue et augmentée, 1999, p. 263.

7. Entre les première et deuxième parties du film, la troupe d'hommes et de chevaux a doublé? : de 6 hommes et 4 chevaux, on est ainsi passé à 10 hommes et 10 chevaux, sans compter Arseniev.

8. Mircea Eliade, article « Chamanisme », Encyclopaedia Universalis, 1985.

9. «L'arbre lui-même a, la plupart du temps, trois niveaux? : les racines qui poussent dans la terre et atteignent les enfers, le tronc qui traverse le niveau des hommes et élève sa couronne vers le ciel inaccessible. »Alexander Eliot, L'Univers fantastique des mythes, VILLE? ?, Editions Les Presses de la Connaissance, 1976. À ce titre, le film de Kurosawa se déroule incontestablement dans le monde des hommes.

10. Un plan similaire se trouve dans Star Wars, épisode IV. Hommage de George Lucas à Kurosawa? ? C'est possible quand on sait l'admiration qu'il porte au cinéaste japonais, dont La Forteresse cachée a inspiré l'intrigue des épisodes de Star Wars.

11. «En descendant le long de la rivière Vangou, nous trouvâmes une ancienne loudéva, clôture spéciale servant à barrer aux fauves l'accès d'un abreuvoir. Pour l'installer, on s'était servi de rompis aussi bien que d'arbres frais, le bois chablis étant consolidé par des piliers qui ne permettaient pas aux bêtes de le disperser de leurs pattes. A quelques endroits, on laisse des passages où sont creusés des trous profonds, habilement masqués par des herbes et des feuilles sèches. La nuit, les cerfs vont à l'eau, mais se heurtent contre la clôture. Voulant la contourner, ils tombent dans ces trous. „Vladimir Arseniev, op.cit., page 119. 
12. Ce chaos est l'œuvre de la nature. Vladimir Arseniev explique dans son livre que les terres ravinées sont souvent la cause de ces chablis.

13. Dans le livre, il est question du sauvetage d'un cerf, qui se déroule dans des conditions beaucoup plus violentes.

14. Walpurgisnacht fait partie du deuxième Faust de Goethe et figure dans la version définitive de 1808. Paris, Editions Aubier, 1976.

15. Chez Goethe, Faust est un savant qui, par orgueil intellectuel, se tourne vers les sciences occultes pour accroître son pouvoir. Certes, Arseniev ne correspond pas à ce portrait. Il n'en demeure pas moins qu'il est l'arpenteur, le mesureur, le géomètre et géographe qui inscrit le monde en chiffres et en coordonnées sur ses cartes.

16. Dans Vivre, un autre Méphisto sert de guide nocturne à Watanabe sans revêtir de fonction maléfique.

17. On peut aussi citer le sapin de Noël musical, décoré de glaçons et d'objets métalliques comme autant de grelots. Cette scène se passe, dans le récit d'Arseniev, dans la nuit du 23 au 24 décembre. Kurosawa la place dans la nuit du Nouvel An, seuil symbolique d'une ère nouvelle.

18. Samuel Beckett, En attendant Godot, Paris, Éditions de Minuit, 1952, p. 17.

19. Si l'onomastique désigne la science des noms propres, et en particulier des noms de personnes, le terme convient ici tout naturellement pour les noms d'arbres...

\section{AUTEUR}

\section{VALÉRIE ZANELLO}

Professeur Agrégé de Lettres Modernes

Doctorante - Laboratoire de Recherche en Audiovisuel

Université de Toulouse II Le Mirailfr 